\title{
Alcoholic hepatitis: should we combine old drugs for better results?
}

\author{
Joaquin Cabezas ${ }^{1} \cdot$ Ramon Bataller $^{1}$
}

Received: 12 July 2016/ Accepted: 30 August 2016/Published online: 15 September 2016

(C) Asian Pacific Association for the Study of the Liver 2016

\begin{abstract}
Alcoholic liver disease (ALD) is the main cause of cirrhosis worldwide [1]. Despite its high prevalence, ALD has received little attention in the liver community and there is a clear lack of programs for early detection and novel targeted therapies for advanced forms. ALD encloses a wide spectrum of features, ranging from simple steatosis, steatohepatitis, and cirrhosis to hepatocellular carcinoma [2]. Patients with underlying ALD and heavy alcohol intake can develop an episode of alcoholic hepatitis (AH), the most severe form of ALD [3]. AH is characterized by an abrupt onset of jaundice and/or liver decompensation. Patients with $\mathrm{AH}$ often show signs of systemic inflammation at admission, and are prone to infections and kidney injury, which can lead to multi-organ dysfunction within a few days [4]. In its severe form, AH can result in 20-50 \% mortality at 3 months. The only available therapies that improve survival are corticosteroids and liver transplantation [5, 6]. The efficacy of pentoxifylline is debatable, based on recent large clinical trials [6]. The efficacy of prednisolone has been shown in meta-analyses of individual data and was partially confirmed in the recent STOPAH trial [6, 7]. Unfortunately, many patients do not respond to prednisolone and performing salvage liver transplantation is not feasible in most centers. Therefore, there is an urgent need to provide these patients with novel targeted therapies. In the last two decades, there have been several attempts to test old and novel drugs in patients with severe $\mathrm{AH}$, ranging from classic drugs [8] to monoclonal antibodies [9] or stimulating factors [10]
\end{abstract}

Ramon Bataller

bataller@med.unc.edu

1 Departments of Medicine and Nutrition, Liver Center, University of North Carolina at Chapel Hill, Chapel Hill, NC 27599, USA to bone marrow transplantation [11]. While some of them have improved short-term mortality, the results have not yet been confirmed in independent cohorts. Since prednisolone consistently improves the 1-month survival in patients with severe $\mathrm{AH}$, a common strategy is to add other drugs that could target some pathogenic driver in this disease such as anti-TNF drugs like Infliximab, which have been tested in combination with prednisolone [9]. While the initial results were encouraging, the usage of a high dose of TNF blocking agents increased the patient's mortality, likely through the increase of severe bacterial infections [12].

One of the hallmark consequences of alcohol abuse is the induction of marked oxidative stress in the liver. Alcohol is known to stimulate reactive oxygen species (ROS) by activating NADPH oxidase and CYP2E1 among other oxidant pathways [13, 14]. Moreover, advanced liver disease is characterized by defective antioxidant systems including hepatocellular content in glutathione (GSH). GSH is an important antioxidant in plants, animals, fungi, and some bacteria. GSH reduces disulfide bonds formed within cytoplasmic proteins to cysteines by serving as an electron donor. Once oxidized, GSH can be reduced back by glutathione reductase, using NADPH as an electron donor. The ratio of reduced glutathione to oxidized glutathione within cells is often used as a measure of cellular oxidative stress [15]. Of note, there are important determinants of $\mathrm{AH}$ other than oxidative stress, including immune cell activation and inflammatory mediators release, intestinal microbiome changes and leaky gut-bacterial translocation, attraction of neutrophils, and release of danger signals [i.e. pathogen-associated molecular patterns (PAMPs) and damage-associated molecular patterns (DAMPs)] [16]. Therefore, it is conceivable that effective drug therapies for $\mathrm{AH}$ should target multiple pathophysiological pathways other than oxidative stress. 
Based on experimental studies indicating that severe ALD is associated with decreased GSH synthesis and resulting oxidative stress [17], Nguyen-Khac et al. performed a large clinical trial using $N$-acetyl cysteine (NAC) (a drug that provides cysteine for glutathione synthesis) in combination with prednisolone [18]. In this important study, NAC administration reduced the development of infections and hepatorenal syndrome and decreased 2-month mortality. This study strongly suggests that replenishing GSH could be beneficial in patients with severe $\mathrm{AH}$ receiving prednisolone. Whether the use of antioxidants alone is able to improve survival in patients with $\mathrm{AH}$ is controversial [19, 20]. While the study by Phillips et al. [19] showed no beneficial effect, a clinical trial by Stewart et al. [20] showed improved short-term outcome. Further studies including end-points other than survival are clearly needed to assess the efficacy of antioxidants in alcoholic liver disease including AH.

An alternative approach to treat conditions associated with depleted GSH is the use of S-adenosylmethionine (SAMe). SAMe, which is well tolerated as an oral presentation, is a co-substrate involved in methyl group transfer and has been shown to increase cellular GSH content in diseases characterized by GSH depletion, such as alcoholic liver disease [21]. SAMe is a precursor for the synthesis of cysteine and thus is required for the generation of GSH [22]. In this issue of Hepatology International, Tkachenko et al. [23] performed a clinical trial adding SAMe, a drug with similar biological effects compared with NAC, to prednisolone. In the current study, 40 patients with severe $\mathrm{AH}$ were randomized to receive prednisolone alone or in combination with SAMe for 1 month, continuing the latter for another month. The primary end-point of the study was 1-month mortality. The initial response, as assessed by the Lille model, was much better in patients receiving SAMe (95 vs. $65 \%$ ). Although there was a trend to reduce 1-month mortality (0 vs. $10 \%$ ), the primary end-point was not reached given the low number of patients. Interestingly, hepatorenal syndrome occurred in $20 \%$ of the prednisolone group, while no cases were registered in the combination group. The results of this interesting study suggest that refilling GSH increases the efficacy of prednisolone in patients with AH.

The results of the current study reveal a crucial role for antioxidant defenses in the pathogenesis of AH. Alcohol abuse is associated with an increased production of ROS and efficient and intact antioxidant systems are essential to protect hepatocytes from cell death [24]. Depletion of GSH, a key antioxidant molecule, could favor the accumulation of ROS and the resulting oxidative stress. It is well known that oxidative stress plays a role in the main pathogenic events in AH. First, it favors hepatocellular death in animals exposed chronically to ethanol, a key feature of AH. Second, it stimulates hepatic stellate cells to produce excessive amounts of collagen and induces the diffusion of hepatic fibrosis [25]. And, finally, it is associated with an increased rate of bacterial infections [26]. An additional potential benefit of antioxidants in the setting of cirrhosis and $\mathrm{AH}$ is the prevention of hepatorenal syndrome. The fact that systemic inflammation precedes and predicts the development of renal failure in patients with AH supports this hypothesis [4]. Finally, antioxidants such as SAMe could be beneficial in patients with alcoholic cirrhosis regardless of the presence of superimposed AH. In these lines, Mato et al. [27] tested the utility of long-term administration of SAMe in patients with alcoholic cirrhosis. This study showed that prolonged treatment SAMe may improve survival in patients with less advanced alcoholic liver cirrhosis, such as Child $\mathrm{A}$ and $\mathrm{B}$ patients.

Although the study by Tkachenko et al. yielded promising results, clinical trials with well-characterized patients with $\mathrm{AH}$ that include a larger number of patients are needed to conclude that SAMe should be indicated in this setting. Moreover, it is possible that longer follow-up periods (up to 3 months) are needed to reach higher mortality rates. Future studies should also measure the degree of oxidative stress in the liver and systemically to identify those patients that would benefit from antioxidant therapies. In this regard, the combination between Silymarin and SAMe has been recently brought to market, and this combination appears to have a promising effect on patients with ALD [28]. Prospective clinical trials in patients with ALD are needed to test this therapeutic approach.

In conclusion, the study by Tkachenko et al. showed that the addition of SAMe to prednisolone improves the shortterm outcome of patients with $\mathrm{AH}$. This study, in conjunction with the previous one showing the beneficial effects of NAC, indicates that targeting oxidative stress might be beneficial to patients with this devastating clinical condition. Based on the available data, the use of antioxidants to treat $\mathrm{AH}$ cannot as yet be strongly recommended. Whether the use of antioxidants (NAC and SAMe) in combination with prednisolone improves survival requires larger clinical trials.

Conflicts of interest Joaquin Cabezas and Ramon Bataller disclose no conflicts.

Financial support This work was supported by the National Institute on Alcohol Abuse and Alcoholism (NIAAA) (1U01AA021908-01 and 1U01AA020821). JC was supported by Juan Rodes Scholarship by AEEH (Asociacion Española para el Estudio del Higado). 


\section{References}

1. WHO. Global status report on alcohol and health 2014. http:// apps.who.int/iris/bitstream/10665/112736/1/9789240692763_eng. pdf?ua $=1$

2. Gao B, Bataller R. Alcoholic liver disease: pathogenesis and new therapeutic targets. Gastroenterology 2011;141(5):1572-1785

3. Lucey MR, Mathurin P, Morgan TR. Alcoholic hepatitis. N Engl J Med 2009;360(26):2758-2769

4. Michelena J, Altamirano J, Abraldes JG, et al. Systemic inflammatory response and serum lipopolysaccharide levels predict multiple organ failure and death in alcoholic hepatitis. Hepatology 2015;62(3):762-772

5. Mathurin P, Moreno C, Samuel D, et al. Early liver transplantation for severe alcoholic hepatitis. $N$ Engl $J$ Med 2011;365(19):1790-1800

6. Thursz MR, Richardson P, Allison M, et al. Prednisolone or pentoxifylline for alcoholic hepatitis. New Engl J Med 2015;372(17):1619-1628

7. Mathurin P, O'Grady J, Carithers RL, et al. Corticosteroids improve short-term survival in patients with severe alcoholic hepatitis: meta-analysis of individual patient data. Gut 2011;60(2):255-260

8. Porter HP, Simon FR, Pope CE 2nd, Volwiler W, Fenster LF. Corticosteroid therapy in severe alcoholic hepatitis. A doubleblind drug trial. N Engl J Med 1971;284(24):1350-1355

9. Spahr L, Rubbia-Brandt L, Frossard JL, et al. Combination of steroids with infliximab or placebo in severe alcoholic hepatitis: a randomized controlled pilot study. J Hepatol 2002;37(4):448-455

10. Singh V, Sharma AK, Narasimhan RL, Bhalla A, Sharma N, Sharma R. Granulocyte colony-stimulating factor in severe alcoholic hepatitis: a randomized pilot study. Am J Gastroenterol 2014;109(9):1417-1423

11. Spahr L, Chalandon Y, Terraz S, et al. Autologous bone marrow mononuclear cell transplantation in patients with decompensated alcoholic liver disease: a randomized controlled trial. PLoS ONE 2013;8(1):e53719

12. Boetticher NC, Peine CJ, Kwo P, et al. A randomized, doubleblinded, placebo-controlled multicenter trial of etanercept in the treatment of alcoholic hepatitis. Gastroenterology 2008;135(6):1953-1960

13. Lu Y, Wu D, Wang X, Ward SC, Cederbaum AI. Chronic alcohol-induced liver injury and oxidant stress are decreased in cytochrome P4502E1 knockout mice and restored in humanized cytochrome P4502E1 knock-in mice. Free Radic Biol Med 2010;49(9):1406-1416

14. Kono H, Rusyn I, Yin M, et al. NADPH oxidase-derived free radicals are key oxidants in alcohol-induced liver disease. J Clin Invest 2000;106(7):867-872
15. Kandar R. The ratio of oxidized and reduced forms of selected antioxidants as a possible marker of oxidative stress in humans. Biomed Chromatogr 2016;30(1):13-28

16. Schnabl B. Liver capsule: Mechanisms of alcoholic hepatitis. Hepatology 2016;64(1):276

17. Hirano T, Kaplowitz N, Tsukamoto H, Kamimura S, FernandezCheca JC. Hepatic mitochondrial glutathione depletion and progression of experimental alcoholic liver disease in rats. Hepatology 1992;16(6):1423-1427

18. Nguyen-Khac E, Thevenot T, Piquet MA, et al. Glucocorticoids plus $\mathrm{N}$-acetylcysteine in severe alcoholic hepatitis. N Engl J Med 2011;365(19): 1781-1789

19. Phillips M, Curtis H, Portmann B, Donaldson N, Bomford A, O'Grady J. Antioxidants versus corticosteroids in the treatment of severe alcoholic hepatitis - a randomised clinical trial. J Hepatol 2006;44(4):784-790

20. Stewart S, Prince M, Bassendine M, et al. A randomized trial of antioxidant therapy alone or with corticosteroids in acute alcoholic hepatitis. J Hepatol 2007;47(2):277-283

21. Anstee QM, Day CP. S-adenosylmethionine (SAMe) therapy in liver disease: a review of current evidence and clinical utility. J Hepatol 2012;57(5):1097-1109

22. Mato JM, Corrales FJ, Lu SC, Avila MA. S-adenosylmethionine: a control switch that regulates liver function. FASEB $\mathrm{J}$ 2002;16(1):15-26

23. Tkachenko P, Maevskaya M, Pavlov A, Komkova I, Pavlov C, Ivashkin V. Prednisolone plus S-adenosil-L-methionine in severe alcoholic hepatitis. Hepatol Int 2016. doi:10.1007/s12072-0169751-4

24. Fernandez-Checa JC, Colell A, Garcia-Ruiz C. S-adenosyl-Lmethionine and mitochondrial reduced glutathione depletion in alcoholic liver disease. Alcohol 2002;27(3):179-183

25. Karaa A, Thompson KJ, McKillop IH, Clemens MG, Schrum LW. S-adenosyl-L-methionine attenuates oxidative stress and hepatic stellate cell activation in an ethanol-LPS-induced fibrotic rat model. Shock 2008;30(2):197-205

26. Artigas A, Wernerman J, Arroyo V, Vincent JL, Levy M. Role of albumin in diseases associated with severe systemic inflammation: Pathophysiologic and clinical evidence in sepsis and in decompensated cirrhosis. J Critic Care 2016;33:62-70

27. Mato JM, Camara J, Fernandez de Paz J, et al. S-adenosylmethionine in alcoholic liver cirrhosis: a randomized, placebo-controlled, double-blind, multicenter clinical trial. J Hepatol 1999;30(6):1081-1089

28. Testino G, Leone S, Ansaldi F, Borro P. Silymarin and S-adenosyl-L-methionine (SAMe): two promising pharmacological agents in case of chronic alcoholic hepathopathy. A review and a point of view. Minerva Gastroenterol Dietol 2013;59(4):341-356 